\title{
Interband optical transitions in spherical nanoheterostructures
}

\author{
V.I. Boichuk, I.V. Bilynskyi, I.O. Shakleina \\ Drohobych Ivan Franko State Pedagogical University \\ 3, Stryiska str., 82100 Drohobych, Lviv Region, Ukraine, \\ e-mail:fizmat@drogobych.net
}

Abstract. A spherical semiconductor nanoheterostructure of cubic symmetry is studied in the paper. Accurate solutions of the Schrödinger equation for $\mathrm{S}_{1 / 2}, \mathrm{P}_{1 / 2}, \mathrm{P}_{3 / 2}, \mathrm{D}_{5 / 2}$, and $\mathrm{D}_{7 / 2}$ states of particles are found in the framework of the Luttinger-Baldereschi-Lipari Hamiltonian and the finite value of the band disruption on the boundaries of the media is ascertained. Specific calculations of the quantum dot radius dependence of the hole state energy were performed for the $\mathrm{GaSb} / \mathrm{AlSb}$ heterostructure. The obtained results were compared to the data obtained using the infinite potential well model as well as the model of simple bands for heavy and light holes. The electron energies and electron wavefunctions were found within the isotropic effective mass model taking into account a discontinuity of the conduction bandgap on the heterostructure boundary. Probabilities of $\mathrm{GaSb} / \mathrm{AlSb}$ heterosystem interband transitions were analyzed using the obtained formulae.

Keywords: heterosystem, quantum dot, state energy.

Manuscript received 02.08.05; accepted for publication 25.10.05.

\section{Introduction}

The heterostructures with quantum dots (QDs) are considered to be very promising as luminescent sources $[1,2]$. In addition, such structures are also of interest from the viewpoint of their physical properties, as the current carriers (electrons, holes) are characterized by the restrictions of their motion over all three dimensions. Therefore, there are many theoretical publications concerning the investigation of electron, hole and exciton spectra in the quantum dots of various configurations [3-6].

Some authors investigating quasi-particle energy states do not take into account the complex structure of the valent band in semiconductors constituting the heterosystem $[5,6]$. They use the effective mass approximation, and the finite value of the band disruption at the boundaries of the media; in addition, the non-uniform change of the quasi-particle effective mass in the intermedium transition is taken into account. One can see from calculations that two latter specifications of the theoretical model are extremely important for small quantum dots (of an exciton radius order).

Another series of theoretical works is based on a sixband model for hole states. The state degeneracy, corresponding to the top of the valent band, is taken into consideration in studying the hole, impurity and exciton states in the nanoheterostructures, particularly in the publications [8-10]. However, they used the infinite potential well model for the calculation of the heterostructure quasi-particle spectra that obviously does not include the direct calculation of the energy bandgap as well as the possibility of the particle to tunnel outside the quantum well.

The purpose of this work is to study how the complex structure of the valent band and the finite value of the band splitting at the interface influence on the hole states. The dependence of various hole states on the quantum dot radius was investigated. The results obtained for different models of hole states and quantum wells were compared. The possibility of the interband optical transitions was analyzed.

\section{Statement of the problem. Basic formulae.}

We consider a semiconductor nanocrystal of spherical shape placed into a semiconductor matrix. The potential energy of a hole inside a QD with a radius $R$ is given by:

$$
V(r)= \begin{cases}U_{0}, & r<R, \\ 0, & r \geq R .\end{cases}
$$

We assume that both QD and matrix semiconductors have cubic symmetry, therefore, their valent bands are quasi-isotropic, and the Luttinger parameters $\gamma_{1}$ and $\gamma_{2}$ are about the same $[11,12]$. In this case, the Hamiltonian of the hole is presented by the following expression 
$\hat{H}=\frac{\gamma_{1}}{2} \hat{p}^{2}-\frac{\mu}{9} R_{0}\left(P^{(2)} J^{(2)}\right)+V(r)$,

where $R_{0}=\frac{e^{4}}{2 \gamma_{1} \hbar^{2} \varepsilon_{0}^{2}}, \mu=\frac{2 \gamma_{2}}{\gamma_{1}}, P^{(2)}$ is a second rank tensor of momentum, $J^{(2)}$ is a second rank tensor of angular momentum that corresponds to the spin $\frac{3}{2}, \varepsilon_{0}$ is the dielectric susceptibility of the semiconductor. The Hamiltonian (2) takes into account that QD and matrix semiconductors have big values of the spin-orbital splitting $\Delta_{\mathrm{so}}$, which is larger than the bandgap at the interface. As seen from [2] when the effective spinorbital interaction is considered, the hole behaviour is described as that of the particle with the spin $\frac{3}{2}$ inside a spherical potential well. Matrix elements of the interaction are not equal to zero only for the states with the spin $\mathrm{L}=0, \pm 2$.

Taking into account that the conservation law is valid for the total angular momentum

$\vec{F}=\vec{L}+\vec{J}$,

the wavefunctions for the first six states can be written as [9]

$\Psi_{h}\left(S_{3 / 2}\right)=f_{0}(r)\left|0, \frac{3}{2}, \frac{3}{2}, F_{z}\right\rangle+g_{0}(r)\left|2, \frac{3}{2}, \frac{3}{2}, F_{z}\right\rangle$,

$\Psi_{h}\left(P_{1 / 2}\right)=f_{1}(r)\left|1, \frac{3}{2}, \frac{1}{2}, F_{z}\right\rangle$,

$\Psi_{h}\left(P_{3} / 2\right)=f_{2}(r)\left|1, \frac{3}{2}, \frac{3}{2}, F_{z}\right\rangle+g_{2}(r)\left|3, \frac{3}{2}, \frac{3}{2}, F_{z}\right\rangle$,

$\Psi_{h}\left(P_{5} / 2\right)=f_{3}(r)\left|1, \frac{3}{2}, \frac{5}{2}, F_{z}\right\rangle+g_{3}(r)\left|3, \frac{3}{2}, \frac{5}{2}, F_{z}\right\rangle$,

$\Psi_{h}\left(D_{5} / 2\right)=f_{4}(r)\left|2, \frac{3}{2}, \frac{5}{2}, F_{z}\right\rangle+g_{4}(r)\left|4, \frac{3}{2}, \frac{5}{2}, F_{z}\right\rangle$,

$\Psi_{h}\left(D_{7} / 2\right)=f_{5}(r)\left|2, \frac{3}{2}, \frac{7}{2}, F_{z}\right\rangle+g_{5}(r)\left|4, \frac{3}{2}, \frac{7}{2}, F_{z}\right\rangle$,

where $\left|L, J, F, F_{z}\right\rangle$ is the eigenfunction of $\hat{\bar{F}}$ operator. The radial functions $f_{i}(r)$ and $g_{i}(r)$ are given as the solutions of the following equations: where coefficients $C_{i}$ are determined by the particle state. For the states under consideration they are equal to:

$\Psi_{h}\left(S_{3 / 2}\right): C_{1}=0, C_{2}=\mu ;$

$\Psi_{h}\left(P_{5}\right): C_{1}=\frac{1}{5} \mu, C_{2}=\frac{2}{5} \sqrt{6} \mu$;

$\Psi_{h}\left(P_{1 / 2}\right): C_{1}=\mu, C_{2}=0 ;$

$\Psi_{h}\left(D_{5 / 2}\right): C_{1}=-\frac{5}{7} \mu, C_{2}=\frac{2}{7} \sqrt{6} \mu ;$

$\Psi_{h}\left(P_{3 / 2}\right): C_{1}=-\frac{4}{5} \mu, C_{2}=\frac{3}{5} \mu$;

$\Psi_{h}\left(D_{7 / 2}\right): C_{1}=\frac{2}{7} \mu, C_{2}=\frac{3}{7} \sqrt{5} \mu$.

To find the energy of the corresponding states, one needs to solve the system of differential equations for the unknown functions $f_{i}$ and $g_{i}(i=0,2,3,4,5)$. The exception is $\Psi_{h}\left(P_{1 / 2}\right)$ state, its wavefunction being expressed as the only one radial function $f_{1}(r)$. The analysis of Eqs (4) shows that the system has accurate solutions, however the functions $f_{i}$ and $g_{i}$ are rather cumbersome and are expressed in terms of the Bessel and McDonald functions. Particularly, the functions $f_{2}(r)$ and $g_{2}(r)$ for the state $\Psi_{h}\left(P_{3 / 2}\right)$ are expressed as:

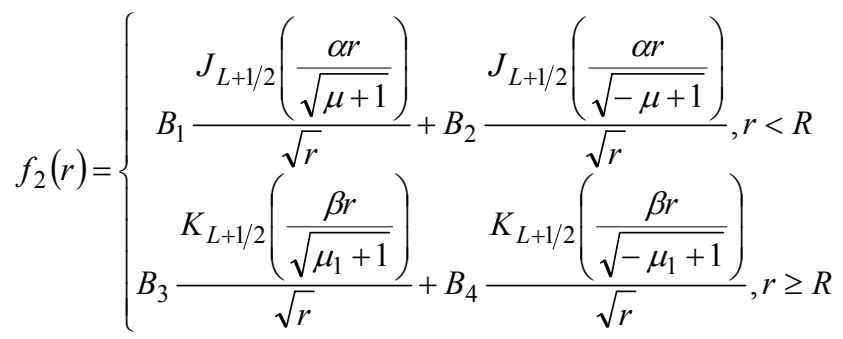

$$
\left(\begin{array}{cc}
-\left(1+C_{1}\right) R_{0}\left(\frac{d^{2}}{d r^{2}}+\frac{2}{r} \frac{d}{d r}-\frac{L(L+1)}{r^{2}}\right)-E+V(r) & C_{2} R_{0}\left(\frac{d^{2}}{d r^{2}}+\frac{(2 L+5)}{r} \frac{d}{d r}-\frac{(L+1)(L+3)}{r^{2}}\right) \\
C_{2} R_{0}\left(\frac{d^{2}}{d r^{2}}-\frac{(2 L+1)}{r} \frac{d}{d r}-\frac{L(L+2)}{r^{2}}\right) & -\left(1-C_{1}\right) R_{0}\left(\frac{d^{2}}{d r^{2}}+\frac{2}{r} \frac{d}{d r}-\frac{(L+2)(L+3)}{r^{2}}\right)-E+V(r)
\end{array}\right)\left(\begin{array}{l}
f_{i} \\
g_{i}
\end{array}\right)=0
$$




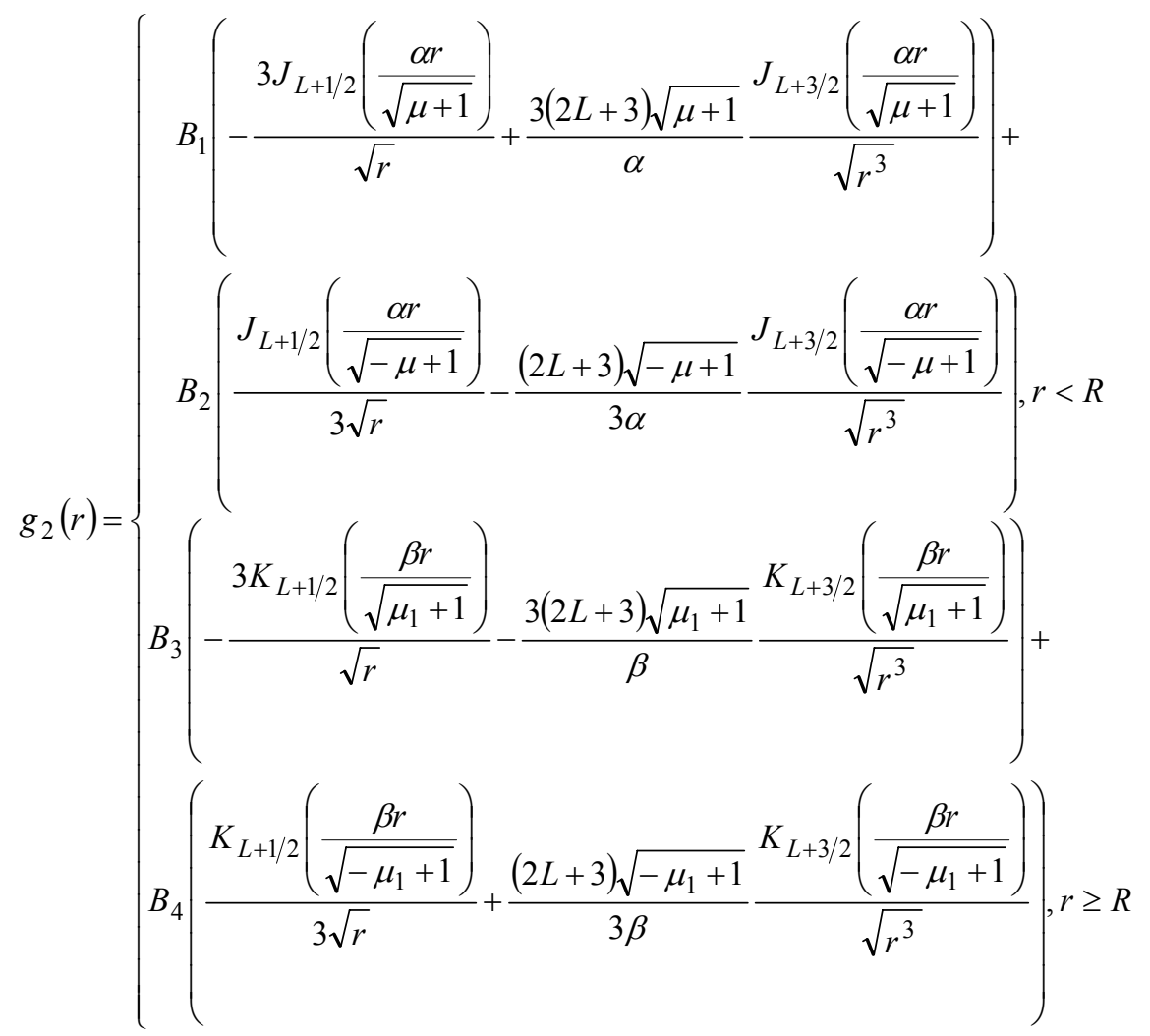

where $J_{l}(r)$ is the first order Bessel function, $K_{l}(r)$ is the McDonald function,

$\alpha=\sqrt{\frac{2 m_{0}}{\hbar^{2} \gamma_{1}^{(1)}}|E|}, \beta=\sqrt{\frac{2 m_{0}}{\hbar^{2} \gamma_{1}^{(2)}}(V-|E|)}$.

The $f_{i}$ and $g_{i}$ functions have to satisfy the standard boundary conditions:

$f_{i}^{(1)}(R)=f_{i}^{(2)}(R), g_{i}^{(1)}(R)=g_{i}^{(2)}(R)$,

$\left.\gamma_{1}^{(1)}\left(1+C_{1}^{(1)}\right) \frac{d f_{i}^{(1)}}{d r}\right|_{r=R}=\left.\gamma_{1}^{(2)}\left(1+C_{1}^{(2)}\right) \frac{d f_{i}^{(2)}}{d r}\right|_{r=R}$,

$\left.\gamma_{1}^{(1)}\left(1-C_{1}^{(1)}\right) \frac{d g_{i}^{(1)}}{d r}\right|_{r=R}=\left.\gamma_{1}^{(2)}\left(1-C_{1}^{(2)}\right) \frac{d g_{i}^{(2)}}{d r}\right|_{r=R}$,

where $f_{i}^{(j)}, g_{i}^{(j)}, C_{i}^{(j)}$ are the wavefunctions and coefficients of $j$-th medium.

For the model of the infinite boundary potential $\left(U_{0}=\infty\right)$, the boundary conditions (7) are simplified: $f_{i}^{(1)}(R)=g_{i}^{(1)}(R)=0$.

From the equations (3)-(7) one can see, that a set of energy levels can be obtained for every value of $L$ and $F$.
For the case of finite $U_{0}$, the number of levels is finite and depends on the radius $R$, meanwhile Eq. (4) has an infinite number of solutions in the case of the infinite potential $U_{0}$.

The degeneracy of the valent band in some points of the Brillouin zone is very often neglected in calculations of the hole and exciton spectra [5]. The hole energy are calculated without considering the valent band complex structure, the different mass of the holes (light and heavy holes) being taken into account only, and the respective laws of dispersion are chosen to be parabolic. The hole energy is found in the same way as the energy of electrons in a simple band. Then, the wavefunction of the particle in a spherical QD is given by

$\Psi(r, \theta, \varphi)=f(r) Y_{l}^{m}(\theta, \varphi)$.

The radial part of the wavefunction $(f(r))$ of $j$-th medium is given by the solution of the equation:

$$
-\frac{\hbar^{2}}{2 m_{j}^{*}}\left(\frac{d^{2}}{d r^{2}}+\frac{2}{r} \frac{d}{d r}\right) f^{(j)}(r)+\frac{\hbar^{2} l(l+1)}{2 m_{j}^{*} r^{2}} f^{(j)}(r)=
$$

$=E f^{(j)}(r), j=1,2$,

$Y_{l}^{m}(\theta, \varphi)$ is a spherical function [13]. The function $f^{(j)}(r)$ satisfies the boundary conditions (7) with the substitution of $\gamma_{1}^{(1)}\left(1+C_{1}^{(j)}\right)$ for $\frac{1}{m_{j}^{*}}$. 


\section{Hole energy spectrum of GaSb/AISb heterostructure}

Specific calculations have been performed for the heterostructure where a spherical nanocrystal $\mathrm{GaSb}$ is placed into the matrix of AlSb crystal. Then we have [11]:

$\gamma_{1}^{(1)}=11.80, \gamma_{1}^{(2)}=4.15, \gamma_{2}^{(1)}=4.03, \gamma_{2}^{(2)}=1.01$,

$U_{0}=0.47 \mathrm{eV}, \Delta_{S 0}^{(1)}=800 \mathrm{meV}, \Delta_{S 0}^{(2)}=750 \mathrm{meV}$,

$m_{h}^{*(1)}=0.23, m_{l}^{*(1)}=0.04, m_{h}^{*(2)}=0.4, m_{l}^{*(2)}=0.12$.

It is convenient to perform calculations using dimensionless quantities, which are introduced as follows

$\alpha=\widetilde{\alpha} \frac{\pi}{a_{0}}, \beta=\widetilde{\beta} \frac{\pi}{a_{0}}, R=\widetilde{R} \frac{\pi}{a_{0}}$,

where $a_{0}$ is the GaSb lattice parameter .

The results of the calculations of the lowest level energy dependence upon the dimensionless radius $\widetilde{R}$ are depicted in Fig. 1. The figure shows that for $L=0(S$ states) the increase of radius $\widetilde{R}$ is accompanied with a monotonic decrease of the hole energy for each value of the radial quantum number $\left(n_{r}\right)$. Comparing the results for the hole ground state energy $\left(n_{r}=1\right)$ in the finite $\left(E_{c}\right)$ potential well model with those for the hole in the infinite $\left(E_{b}\right)$ potential well model, one obtains that $E_{c}<E_{b}$. These results are entirely explained by the Heisenberg uncertainty principle. For the numerical data, the calculations show that for the given heterosystem in the region of $\widetilde{R}>60$ the relative uncertainty is

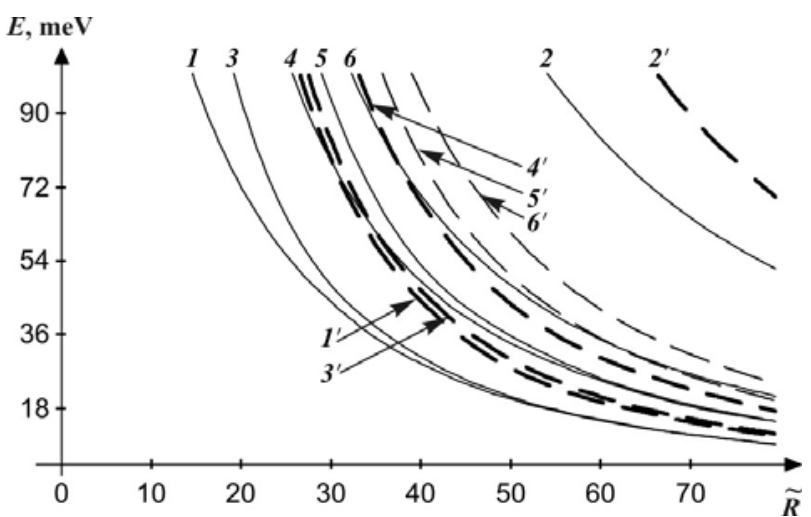

Fig. 1. The lowest level $\left(n_{r}=1\right)$ hole energy dependence upon the dimensionless nanocrystal radius $\widetilde{R}$. The solid line shows the energy obtained in the model of the finite potential well $\left(E_{c}\right)$, the dashed line refers to the model of the infinite potential well $\left(E_{b}\right)$ : Curves $1,1^{\prime}-L=0$ (state $\left.S_{3 / 2}\right) ; 2,2^{\prime}-L=1$ (state $\left.P_{1 / 2}\right) ; 3,3^{\prime}-L=1$ (state $\left.P_{3 / 2}\right) ; 4,5^{\prime}-L=2$ (state $\left.D_{5 / 2}\right) ; 5,4^{\prime}$ $L=1\left(\right.$ state $\left.P_{5 / 2}\right) ; 6,6^{\prime}-L=2\left(\right.$ state $\left.D_{7 / 2}\right)$.
$\eta=\frac{E_{b}-E_{c}}{E_{b}} \leq 22 \%$

For $\widetilde{R}=40, \quad \eta=34.6 \%, \quad$ but for $\widetilde{R}=20$, $\eta=48.4 \%$. The values of the energy of the excited states $\left(n_{r}=2,3, \ldots\right)$, calculated within these models show even larger discrepancy. Even though we obtain a finite number of bound states for $U_{0}=0.47 \mathrm{eV}$, and an infinite number for the infinite well model, the relative uncertainty at given $\widetilde{R}$ increases in comparison to that for $n_{r}=1$, i.e., for $n_{r}=2$ at $\widetilde{R}=40, \eta=35.5 \%$, and for $\widetilde{R}=20, \eta=59.3 \%$.

Let us compare the values of the energy $E_{c}$ with those calculated using the formula (9), which does not include the complex structure of the valent band. The results of the energy calculations for the particles with effective masses $m_{h}^{*}$ and $m_{l}^{*}$ are presented in Fig. 2. The figure shows a good agreement between the energy results for the hole with $m_{h}^{*}$ in the region $\widetilde{R}>20$ (calculated using the equation (9)) and the energy of the particle with $L=0, S=3 / 2$ and $n_{r}=1$ (calculated using the equations (4) and (6)). For $\widetilde{R}<20$, the decrease of $\widetilde{R}$ leads to an abrupt increase of the discrepancy between the corresponding values of energies. The energy of the particle with the effective mass $m_{l}^{*}$ is much larger for all values of $\widetilde{R}$, and it can be compared with the excited states of the hole for $n_{r}=3$. It should be mentioned that the number of states at given $\widetilde{R}$ is also finite in this approach. According to (4), the bound state spectrum is richer than that obtained from (9) for particles with two effective masses. Particularly,

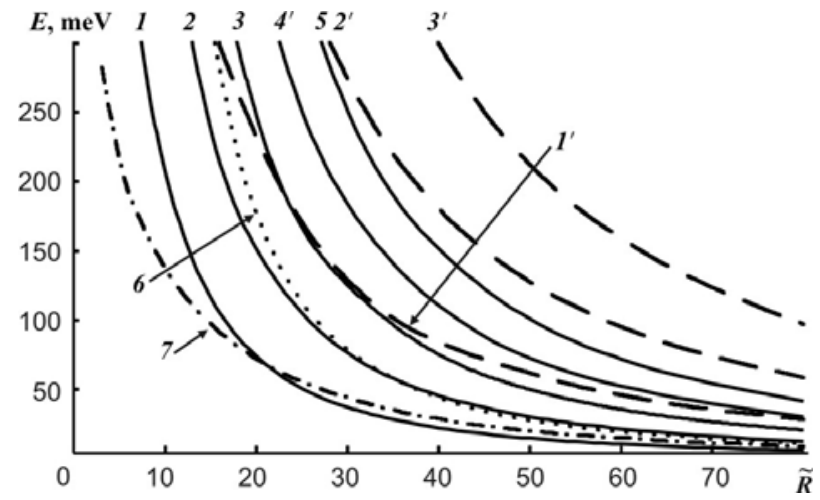

Fig. 2. The $\widetilde{R}$ dimensionless nanocrystal radius dependence of the hole energy $E$. A solid line shows the energy obtained in the model of the finite potential well according to formula (9) for the heavy hole $\left(m_{h}^{*}\right)$, a dashed line refers to the light hole $\left(m_{l}^{*}\right)$, dotted and dash-dotted lines correspond to formula (4): curves $1,1^{\prime}-l=0$ ( $s$-state); $2,2^{\prime}-l=1$ ( $p$-state); $3,3^{\prime}-l=2$ $(d$-state) $44-l=3(f$-state $) ; 5-l=4$ ( $g$-state) $; 6-L=0$ (state $\left.S_{3 / 2}\right) ; 7-L=0$ (state $S_{3 / 2}$ ) in the infinite model. 
according to (4) at $\widetilde{R}=5$, we obtain one bound state with the energy $E_{c}=214 \mathrm{meV}$, while using the equation (9) we also obtain one root for the particle with a larger mass, but its energy is $E=427 \mathrm{meV}$. For $\widetilde{R}=30$, the first approach gives four roots for the bound states (44, 88,176 and $316 \mathrm{meV}$ ), meanwhile, the second approach gives only one root for the lighter particle $(129 \mathrm{meV})$ and three roots for the heavier one $(27,149$, and $335 \mathrm{meV}$ ). For $\widetilde{R}=80$, from Eq. (4) we obtain 14 bound states and either three or eight roots for the light and heavy holes, respectively.

Our analysis of the $E_{c}=E_{c}(\tilde{R})$ dependence shows that the curvature of the graphs depends on the values of $\widetilde{R}$, which is easy to see from Fig. 3, where $\widetilde{R}$ is a dependence of $\widetilde{R}^{2} E_{c}(\widetilde{R})$ for different states is presented. The figure shows that at certain $\widetilde{R}$ the function $\widetilde{R}^{2} E_{c}(\widetilde{R})$ is constant and almost does not depend on $\widetilde{R}$. This means that the function $E_{c}(\tilde{R})$ itself is a hyperbola of const $/ \widetilde{R}^{2}$ type. At small $\widetilde{R}$, this function has $1 / R^{\alpha}$ dependence on $\widetilde{R}$, where $\alpha<2$.

\section{Probabilities of optical transitions}

Let us consider optical transitions from the valent to conduction band for the given heterostructure. The hole wavefunction $\left|L \frac{3}{2} F F_{z}\right\rangle$ in Eq. (3) can be written in the form of the expansion $[9,13]$

$$
\left|L \frac{3}{2} F F_{z}\right\rangle=\sum_{M_{1} M_{2}}\left|L M_{1}\right\rangle 3 / 2\left|M_{2}\right\rangle\left(L 3 / 2 M_{1} M_{2} \mid F F_{z}\right),
$$

where $\left|L M_{1}\right\rangle$ is the eigenvectror of $\hat{L}^{2}$ operator:

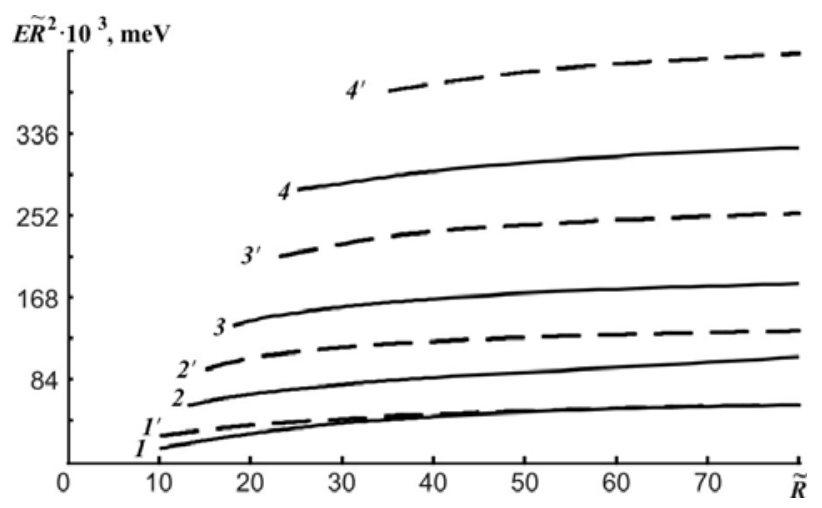

Fig. 3. The $\widetilde{R}$ dependence of $E \widetilde{R}^{2}$ for states $S_{3 / 2}(L=0)$ shown by a solid line, $P_{3 / 2}(L=1)$ by a dashed line: curves 1 , $1^{\prime}-n_{r}=1 ; 2,2^{\prime}-n_{r}=2 ; 3,3^{\prime}-n_{r}=3 ; 4,4^{\prime}-n_{r}=4$.
$L^{2}\left|L M_{1}\right\rangle=\hbar^{2} L(L+1)\left|L M_{1}\right\rangle$,

$\left|3 / 2 M_{2}\right\rangle\left(M_{2}= \pm 1 / 2, \pm 3 / 2\right)$ is the Bloch vector of the particle state with the spin $J=3 / 2$, which corresponds to the fourfold degenerated valent band $\Gamma_{8}$ :

$$
\begin{aligned}
& \left|\frac{3}{2} \frac{3}{2}\right\rangle=\frac{1}{\sqrt{2}}(X+i Y) \uparrow,\left|\frac{3}{2}-\frac{3}{2}\right\rangle=\frac{i}{\sqrt{2}}(X-i Y) \downarrow \\
& \left|\frac{3}{2} \frac{1}{2}\right\rangle=\frac{1}{\sqrt{6}}[(X+i Y) \downarrow-2 Z \uparrow], \\
& \left|\frac{3}{2}-\frac{1}{2}\right\rangle=\frac{i}{\sqrt{6}}[-(X-i Y) \uparrow+2 Z \downarrow],
\end{aligned}
$$

$\left(L 3 / 2 M_{1} M_{2} \mid F F_{z}\right)$ are the Klebsh-Gordon coefficients. The electron wavefunction is presented in a simpler form:

$\Psi_{e}=f_{e}(r)|\operatorname{lm}\rangle|S \sigma\rangle$,

where $f_{e}(r)$ and $|l m\rangle$ are the solutions of the equations (9) and (11), respectively, and $|S \sigma\rangle$ is the Bloch state vector of the electron at the conduction band bottom with $S_{z}=\hbar \sigma($ where $\sigma= \pm 1 / 2)$ as the $z$-component of its spin:

$\left|S \frac{1}{2}\right\rangle=i S \uparrow,\left|S-\frac{1}{2}\right\rangle=i S \downarrow$.

Once having the electron wavefunctions in the valent and conduction bands, one can determine the probability of the interband transition accompanied with creation or absorption of a photon. It is known [9] that the probability is expressed through a square modulus of the matrix element of the momentum operator:

$P_{e h}=\left\langle\Psi_{e}|\vec{e} \hat{\bar{p}}| \Psi_{h}\right\rangle$,

where $\vec{e}$ is a light polarization vector.

As we can see from (3), the function $\Psi_{h}$ has two terms (except for $\Psi_{h}\left(P_{1 / 2}\right)$ ), the angular parts of which differ by the value of the orbital quantum number $L$. Thus, let us consider the angular part of the matrix element (13):

$$
\begin{aligned}
& \sum_{M_{1} M_{2}}\left\langle\operatorname{lm} \mid L M_{1}\right\rangle\left\langle S \sigma|\vec{e} \hat{\bar{p}}| 3 / 2 M_{2}\right\rangle\left(L 3 / 2 M_{1} M_{2} \mid F F_{z}\right)= \\
& \sum_{M_{2}}\left\langle S \sigma|\vec{e} \hat{\bar{p}}| 3 / 2 M_{2}\right\rangle\left(l 3 / 2 m M_{2} \mid F F_{z}\right) \equiv\left\langle\operatorname{lm}|\vec{e} \hat{\bar{p}}| F F_{z}\right\rangle
\end{aligned}
$$

In (14), we took into account the orthogonality of spherical functions: 
$\left\langle l m \mid L M_{1}\right\rangle=\delta_{l, L} \delta_{m, M_{1}}$.

If we take (14) into account, $P_{e h}$ will be expressed through one of the two terms of the function $\Psi_{h}$, because the other one will be orthogonal to the wavefunction of the electron in the conduction band:

$$
P_{e h}=\int d r r^{2} f_{e}^{*}(r) f_{h}(r)\left\langle\operatorname{lm}|\vec{e} \hat{\bar{p}}| F F_{z}\right\rangle=I\left\langle l m|\vec{e} \hat{\bar{p}}| F F_{z}\right\rangle .
$$

For a crystal of cubic configuration and linearly polarized light it is convenient to expand the scalar product $\vec{e} \hat{\bar{p}}$ in the form of sum

$\vec{e} \hat{\bar{p}}=e_{x} \hat{p}_{x}+e_{y} \hat{p}_{y}+e_{z} \hat{p}_{z}$.

Let us determine the contribution of the angular part of the matrix element to the probability of interband transitions for various light polarizations. We take into account the properties of Klebsh-Gordon's coefficients. For the conduction band electron with the "up" spin and "down" spin we get the following non-zero matrix elements

$$
\begin{aligned}
& \left|\left\langle 00\left|p_{x}\right| \frac{3}{2} \frac{3}{2}\right\rangle\right|^{2}=\left|\left\langle S \uparrow\left|\hat{p}_{x}\right| \frac{3}{2} \frac{3}{2}\right\rangle\right|^{2}=\frac{1}{2}\left|\left\langle S\left|\hat{p}_{x}\right| x\right\rangle\right|^{2} \equiv \frac{1}{2} P^{2}, \\
& \left|\left\langle 00\left|p_{x}\right| \frac{3}{2}-\frac{3}{2}\right\rangle\right|^{2}=\left|\left\langle S \downarrow\left|\hat{p}_{x}\right| \frac{3}{2}-\frac{3}{2}\right\rangle\right|^{2}=\frac{1}{2} P^{2}, \\
& \left|\left\langle 00\left|p_{x}\right| \frac{3}{2}-\frac{1}{2}\right\rangle\right|^{2}=\left|\left\langle S \uparrow\left|\hat{p}_{x}\right| \frac{3}{2}-\frac{1}{2}\right\rangle\right|^{2}=\frac{P^{2}}{6}, \\
& \left|\left\langle 00\left|p_{x}\right| \frac{3}{2} \frac{1}{2}\right\rangle\right|^{2}=\left|\left\langle S \downarrow\left|\hat{p}_{x}\right| \frac{3}{2} \frac{1}{2}\right\rangle\right|^{2}=\frac{P^{2}}{6} .
\end{aligned}
$$

The total probability of light polarization along the OX axis is $\frac{4}{3} P^{2}$. We obtain the same values of matrix elements also in the OY direction. The probabilities determined by the operator $p_{z}$ are as follows

$$
\begin{aligned}
& \left|\left\langle 00\left|p_{z}\right| \frac{3}{2} \frac{1}{2}\right\rangle\right|^{2}=\left|\left\langle S \uparrow\left|\hat{p}_{z}\right| \frac{3}{2} \frac{1}{2}\right\rangle\right|^{2}=\frac{2}{3}\left|\left\langle S\left|\hat{p}_{z}\right| z\right\rangle\right|^{2} \equiv \frac{2}{3} P^{2}, \\
& \left|\left\langle 00\left|p_{z}\right| \frac{3}{2}-\frac{1}{2}\right\rangle\right|^{2}=\left|\left\langle S \downarrow\left|\hat{p}_{z}\right| \frac{3}{2}-\frac{1}{2}\right\rangle\right|^{2}=\frac{2}{3} P^{2} .
\end{aligned}
$$

The sum of these two probabilities is also equal to $\frac{4}{3} P^{2}$, as it is expected in a cubic crystal since all directions in space are equal.

Calculations of the overlapping integral of the radial wavefunctions $I$ for the finite and infinite potential of QW show that the nanocrystal radius dependence of $I$ strongly depends on the chosen model of the potential. Fig. 4 shows, for $V_{0}=\infty$, the overlapping integral does not depend on $\widetilde{R}$, even though its value depends on the type of transition. Particularly, for $s$ transition, $n_{r}=1-$ $S_{3 / 2}, n_{r}=1$, we obtain $I=0.595$, while for $s$ transition, $n_{r}=2-S_{3 / 2}, n_{r}=1$, we have $I=0.51$, and for $p$

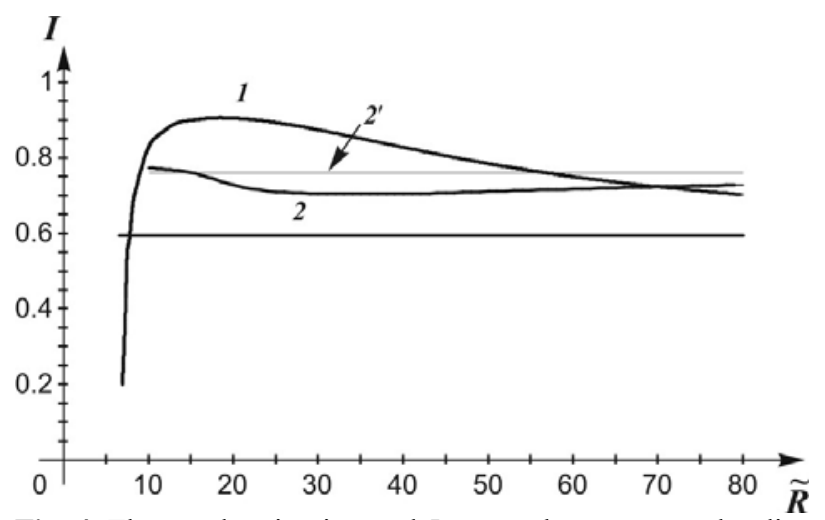

Fig. 4. The overlapping integral $I$ versus the nanocrystal radius $\widetilde{R}$. The straight lines stand for the choice of $V_{0}=\infty$, curves refer to the model of finite $V_{0} \cdot 1,1^{\prime}$ correspond to the transition $1,1^{\prime}-\mathrm{s}, n_{r}=1-\mathrm{S}_{3 / 2}, n_{r}=1,2,2^{\prime}-\mathrm{s}, n_{r}=2-\mathrm{S}_{3 / 2}$.

transition, $n_{r}=1-P_{3 / 2}, n_{r}=1, I=0.95$ is obtained. One can see that the possibility of both $\Delta n_{r}=0$, and $\Delta n_{r} \neq 0$ transitions is obtained. The received results are in good agreement with the data $[9,12]$, where other heterostructures were considered.

More interesting results are obtained for the finite $V_{0}$ model. In this case, the overlapping integral $I$ depends on $\widetilde{R}$. The function $I=I(\widetilde{R})$ depends on the type of transition. For large $\widetilde{R}$, for each transition the functions $I=I(\widetilde{R})$ asymptotically tends to their values as $V_{0} \rightarrow \infty$. Another common feature of the mentioned functions is that they decrease monotonically for small $\widetilde{R}$. Therefore, the consideration of the finite value of the band splitting at the boundaries of the heterostructure results in decreasing the possibility of optical transitions with decreasing the $\mathrm{QD}$ radius.

Therefore, in the paper the energy of hole states of $\mathrm{GaSb} / \mathrm{AlSb}$ spherical nanoheterostructures is found by using the isotropic valent band approximation for the Luttinger Hamiltonian with the consideration of the finite band disruption. It is shown that in the range of small radii the consideration of both degeneracy of hole states and the finiteness of a quantum well essentially influences numerical values of the hole energy.

On the basis of the formulae obtained for the wavefunctions of hole states, the probabilities of interband transitions have been analyzed in absorption and illumination of the mentioned heterostructures.

\section{References}

1. M. Bissiri, G. Baldassari H.v.H., M. Capizzi, V.M. Fomin, V.N. Gladilin and O.C. Vreese // Phys. status solidi (b) 224(3), p.639-642 (2001).

2. V.A. Burdov // Fizika Tverdogo Tela 43(6), p. 11101115 (2001) (in Russian). 
3. V.I. Boichuk, R.Yu. Kubay // Ibid. 43 (2), p. 226-232 (2001) (in Russian).

4. M.V. Tkach // The 2-nd Ukrainian Scientific Conference for Physics of Semiconductors. Abstracts. V.1. Chernivtsi - Vyzhnytsya, 20-24 September, p. 5 (in Russian).

5. M.V. Tkach, A.V. Makhanets', P.G. Zegrya // Fizika $i$ Tekhnika Poluprovodnikov 36(5), p. 543-549 (2002) (in Russian).

6. M.V. Tkach, V.A. Golovatsky, O.A. Voytsekhovskaya // Ibid. 34(5), p. 602-606 (2000) (in Russian).

7. J.M. Luttinger and W. Kohn // Phys. Rev. 97(4), p. 869-888 (1955).
8. A1.L. Efros, A.L. Efros // Fizika $i$ Tekhnika Poluprovodnikov 16(7), p. 1209-1214 (1982) (in Russian).

9. Jian-Bai Xia // Phys. Rev. B 40(12), p. 8500-8507 (1989).

10. V.A. Zerova, L.Ye. Vorobyov, G.P. Zegrya // Fizika $i$ Tekhnika Poluprovodnikov 38(6), p. 716-722 (2004) (in Russian).

11. A. Baldereshi, Nunzio O. Lipari // Phys. Rev. B 8(6), p. 2697-2709 (1973).

12. Al.L. Efros // Phys. Rev. B 46(12), p. 7448-7458 (1992-II).

13. A.S. Davydov, Quantum mechanics. Nauka, Moscow (1978). 